\title{
Regulation of Transcription Activity of MAKR4 in Arabidopsis thaliana L.
}

\author{
Anastasia Korosteleva \\ Novosibirsk State University \\ Novosibirsk, Russia \\ kartzeva.kar@gmail.com
}

\author{
Daria Novikova \\ Institute of Cytology and Genetics \\ SB RAS \\ Novosibirsk, Russia \\ da6ik777@gmail.com
}

\author{
Victoria Mironova \\ Institute of Cytology and Genetics \\ SB RAS, Novosibirsk, Russia \\ kviki@bionet.ns.ru
}

\begin{abstract}
Auxin has a crucial influence on genes expression. In this work, we show that transcriptional activity of an auxin-sensitive gene MAKR4 (MEMBRANE ASSOSIATED KINASE REGULATOR 4) depends on the composite elements in its upstream regulatory region. Analysis of such elements could clarify the understanding of the regulation of this gene and other auxin-sensitive genes.
\end{abstract}

Keywords - MEMBRANE ASSOCIATED KINASE REGULATORS, lateral roots, auxin, Arabidopsis thaliana

\section{Introduction}

Genes of MEMBRANE ASSOCIATED KINASE REGULATORS family play an important role in plant morphogenesis. One of the family members, MAKR4, is implicated in lateral root formation. MAKR4 was shown to convert the prebranch sites into regular lateral organs [1]. It is assumed that the accumulation of MAKR4 on the plasma membrane of pericycle cells triggers the processes which leads to the formation of a lateral root.

In the promoter region of MAKR4 auxin-associated ciselements were predicted (Auxin Response Elements, AuxRE). Some of these were predicted to bind ARF transcription factors (AUXIN RESPONSE FACTORS) and some transcription factors from other families (non-ARF). Possibly, these proteins together provide auxin response of MAKR4. ARFs are known to form homodimers and heterodimers with each other and transcription factors from other families $[2,3]$. The interaction of ARFs and potential partner-proteins in regulation of transcriptional response to auxin could explain formation of the complex MAKR4 expression pattern in Arabidopsis thaliana.

\section{Results}

The predicted AuxREs in MAKR4 upstream regulatory region were annotated with a variety of bioinformatics tools.
We obtained homozygous reporter lines of Arabidopsis thaliana plants with GFP expressed under the intact promoter of $M A K R 4$, as well as under promoter versions with mutated predicted cis-elements.

The expression pattern of GFP was studied with fluorescence microscopy in the control plants and after threehour auxin treatment. Arabidopsis lines with an intact MAKR4 promoter had a significant increase in the expression level of GFP. In Arabidopsis lines with mutated predicted ciselements (AuxRE or non-AuxRE), a significant increase in the expression of GFP did not occur. Lines with two or three ciselements mutated at the same time had a low level of GFP expression and, thus, did not posess response to auxin.

Analysed cis-elements in MAKR4 upstream regulatory region have an impact on the auxin-mediated gene expression.

\section{Acknowledgment}

This work was supported by RFBR 19-44-543006.

\section{References}

[1] Xuan, W., Audenaert, D., Parizot, B., Möller, B. K., Njo, M. F., De Rybel, B., ... \& Beeckman, T. (2015). Root cap-derived auxin prepatterns the longitudinal axis of the Arabidopsis root. Current Biology, 25(10), 1381-1388.

[2] Boer, D. R., Freire-Rios, A., van den Berg, W. A., Saaki, T., Manfield, I. W., Kepinski, S., ... \& Weijers, D. (2014). Structural basis for DNA binding specificity by the auxin-dependent ARF transcription factors. Cell, 156(3), 577-589.

[3] Simonini, S., Deb, J., Moubayidin, L., Stephenson, P., Valluru, M., Freire-Rios, A., ... \& Østergaard, L. (2016). A noncanonical auxinsensing mechanism is required for organ morphogenesis in Arabidopsis. Genes \& development, 30(20), 2286-2296. 